\title{
EVALUATION OF DIFFUSION-WEIGHTED IMAGING AND APPARENT DIFFUSION COEFFICIENT MAPPING USING DIFFERENT $B$-VALUES FOR MAGNETIC RESONANCE- GUIDED FOCUSED ULTRASOUND SURGERY: A PRELIMINARY STUDY FOR UTERINE FIBROID AND ADENOMYOMA
}

\author{
Panicker $A^{1}$, Abdullah $\mathrm{BJ}^{1}$, Yaakup $\mathrm{NA}^{1}$, Wong $\mathrm{YH}^{1}$, Yeong $\mathrm{CH}^{1}$, Omar $\mathrm{SZ}^{2}$, Vijaynanthan $\mathrm{A}^{1}$ \\ ${ }^{1}$ Department of Biomedical Imaging, Faculty of Medicine, University of Malaya, 50603 Kuala Lumpur, Malaysia \\ ${ }^{2}$ Department of Obstetrics and Gynaecology, Faculty of Medicine, University of Malaya, 50603 Kuala Lumpur, Malaysia
}

\section{Correspondence:}

Dr. Yeong Chai Hong

Department of Biomedical Imaging, Faculty of Medicine, University of Malaya, 50603 Kuala Lumpur, Malaysia. Email: chyeong@um.edu.my

Tel: +60167016875

\begin{abstract}
The study was taken to assess the feasibility of diffusion-weighted imaging (DWI) and apparent diffusion coefficient (ADC) mapping using different $b$-values for magnetic resonance-guided focused ultrasound (MRgFUS) treatment of uterine fibroid and adenomyoma.
\end{abstract}

The contrast-enhanced T1-weighted image (cT1WI) as well as DWIs and ADC maps of different $b$-values (i.e. 200,600 and $800 \mathrm{~s} / \mathrm{mm}^{2}$ ) were obtained from nine fibroid and five adenomyoma patients, immediately after, and 12 months after MRgFUS treatment. The image contrast score, non-perfused volume (NPV) and NPV ratio obtained were compared to determine the feasibility of DWI and ADC mapping for MRgFUS treatment outcome evaluation.

\begin{abstract}
Our finding showed that immediately after MRgFUS treatment, the DWI acquired using $200 \mathrm{~s} / \mathrm{mm}^{2} b$-value gave the highest image contrast score among all other $b$-values. The NPV calculated from DWI of $200 \mathrm{~s} /$ $\mathrm{mm}^{2}$ showed the best correlation $\left(R^{2}=0.938\right)$ with post-contrast NPV. At 12 months follow-up, there was no specific $b$-value considered as significantly superior to others in terms of image contrast. However, the NPVs and NPV ratios obtained from all DWIs and ADC maps of different $b$-values were in good agreement with the post-contrast NPV and NPV ratio.
\end{abstract}

We observed that the DWI, particularly obtained with a low $b$-value (i.e. $200 \mathrm{~s} / \mathrm{mm}^{2}$ ), is feasible for delineation and quantitative volumetric evaluation of the ablated region immediately after the MRgFUS treatment. At 12 months follow-up, both DWIs and ADC maps are feasible for NPV and NPV ratio calculation.

Keywords: Apparent Diffusion Coefficient (ADC), Diffusion-Weighted Imaging (DWI), Magnetic ResonanceGuided Focused Ultrasound (Mrgfus), Magnetic Resonance Imaging (MRI), Uterine Fibroid And Adenomyoma

\section{Introduction}

Uterine fibroids and adenomyoma are benign pathological diseases that have affected women in the reproductive age group. These diseases have caused significant disability and morbidity in patients and constitute a major source of public healthcare cost worldwide $(1,2)$. Numerous therapeutic strategies such as hormone therapy, drug therapy, surgical intervention, uterine artery embolization and magnetic resonance-guided focused ultrasound
(MRgFUS) ablation, are available to treat uterine fibroids and adenomyoma (2-6). The MRgFUS is proven to be a feasible, safe, efficacious treatment for uterine fibroids and adenomyoma (7-14) by inducing coagulative necrosis, size reduction and functional loss of the lesion $(7,15)$. The treatment outcome of MRgFUS ablation is normally assessed by contrast-enhanced T1-weighted image (cT1WI) with the use of gadolinium-based magnetic resonance contrast agent. 
Although cT1WI has been widely accepted as the gold standard for treatment outcome evaluation, several limitations are found with the technique. The application of contrast enhanced magnetic resonance imaging (MRI) could be limited due to patient allergies towards gadolinium. Further, overestimation of ablated volume may occur when there is a transient reduction of vascularity in non-treated fibroid tissue due to vessel spasm or other factors (16). Besides, the unknown hazardous potential and side effects of heating the contrast agent have limited the peri-procedural contrast enhanced imaging. Due to the same reason, additional treatment would need to be scheduled in the cases of incomplete ablation as MRgFUS is not recommended to be applied immediately after the contrast enhanced MRI scan. In view of the above, the method which could be used for peri- and post-procedural treatment outcome evaluation is therefore favourable.

The diffusion-weighted imaging (DWI) may be feasible to serve as a "functional parameter" to evaluate the periand post-procedural treatment outcome by displaying the contrast based on the differences in the water molecules diffusivity within intra- and extracellular spaces. Various studies have reported the use of DWI and apparent diffusion coefficient (ADC) mapping in monitoring and assessing the treatment outcomes of MRgFUS of the uterine fibroids and uterine artery embolization (17-23). However, the correlation between the quantitative volume measurements of the ablated fibroid or adenomyoma obtained from DWI or ADC map and post-contrast nonperfused volume (NPV) obtained from standard cT1WI are not known to be performed previously.

Hence, this study was taken to assess the feasibility of DWI and ADC map in quantitative treatment volume measurement, immediately after MRgFUS treatment and at 12 months follow-up. In particular, our aim was to investigate which image (DWI or ADC map) and which $b$-value $\left(200,600\right.$ or $\left.800 \mathrm{~s} / \mathrm{mm}^{2}\right)$ would provide a better correlation to the standard CT1WI NPV results.

\section{Methods}

\section{Patient population}

Patients who have been diagnosed with uterine fibroid or adenomyoma were recruited in this study. The inclusion and exclusion criteria are as follows:

\section{Inclusion Criteria:}

- $\quad$ Premenopausal women older than 18 years or peri-menopausal women with a normal cervical smear and symptomatic fibroid or adenomyosis disease.

- $\quad$ Patients with confirmed fibroid disease or adenomyoma by clinical examination and deemed suitable for MRgFUS treatment.

\section{Exclusion Criteria:}

- Menstruating, pregnant or lactating women.

- $\quad$ Patients having contraindication to standard MR imaging, IV sedation or IV gadolinium chelate.

- $\quad$ Patients with suspected or confirmed uterine malignancy, other pelvic diseases or major systemic diseases.

- $\quad$ Patients showing extensive lower abdominal wall scarring which increases the risk of pain or skin burns.

Medical ethics approval was obtained from the Medical Ethics Committee, University of Malaya Medical Center (UMMC). All patients were counselled on the safety and risks of MRI examination and MRgFUS treatment procedure. Written informed consent about the treatment, the use of conscious sedation and gadolinium contrast agent was obtained from all patients.

\section{Imaging protocol}

MRI scanning during pre-treatment, immediately after MRgFUS treatment and at 12 months follow-up, were performed on a 1.5 Tesla MRI scanner (Signa HDxt, General Electric Medical Systems, Wisconsin, USA). The T2-weighted (T2WI) fast spin echo sequences [repetition time (TR): 3200 - 4000 ms; echo time (TE): 90 - 110 ms, echo train length (ELT): 24], in sagittal and axial planes, were acquired relative to the orientation of the uterine cavity in 5 $\mathrm{mm}$ slices with $1 \mathrm{~mm}$ spacing. Then, the axial fat-saturated three dimensional (3D) gradient echo T1WI sequence (TR: 5.32; TE: $2.53 \mathrm{~ms}$ ) and axial spin-echo T1WI sequence (TR: 536; TE: $11 \mathrm{~ms}$ ) were acquired followed by additional axial spin-echo echo-planar diffusion-weighted imaging (SE-EPIDWI) sequence (TR: 8000; TE: 69 ms; $b$-values: 200, 600 and $800 \mathrm{~s} / \mathrm{mm}^{2}$ ). The ADC maps were constructed from the DWI data using the dedicated software. Following DWI, the contrast-enhanced imaging was performed using axial 3D gradient echo T1WI sequence after administration of $10 \mathrm{ml}$ intravenous bolus of Gadopentetate Dimeglumine (Magnevist; Bayer HealthCare Pharmaceuticals Inc., Berlin, Germany).

Both pre- and post-contrast enhanced T1WI were used to determine the lesion's viability while T2WI was used to measure and calculate the volume of the treatable fibroid or adenomyoma by assuming the volume $(v)$ of a prolate ellipsoid using the following equation:

$v=\frac{4}{3} \pi \times \frac{\mathrm{L}}{2} \times \frac{\mathrm{W}}{2} \times \frac{\mathrm{AP}}{2}$

where L, W and AP represent the length, width and anteroposterior length of the lesion in $\mathrm{cm}$, respectively. The DWIs and ADC maps were used for quantitative volume assessment of the NPV. 


\section{Magnetic resonance-guided focused ultrasound treatment}

The MRgFUS treatment of all patients was performed in the prone position using the MRgFUS system (ExAblate ${ }^{\circledR}$ 2000, InSightec, Israel) following the standard procedure as described earlier (24). Patients were under conscious sedation and given optimized pain relief with the vital signs such as blood pressure and pulse rate being monitored throughout the procedure. The occurrence of any adverse events such as skin burns, bowel injury and sciatica was recorded during or immediately after the procedure. The patients were discharged after a minimum of 2 hours' observation or until the effects of conscious sedation wore off.

\section{Patient follow-up}

Patients were followed up at 12 months after MRgFUS procedure by a repeated routine pelvic MRI following the same imaging protocol as mentioned above.

\section{Image analysis}

The image contrast between the ablated and non-ablated regions on CT1WIs, DWIs and ADC maps, both immediately after the treatment and at 12 months follow-up, were evaluated by two senior radiologists using the scoring system below:

- $\quad$ Score 0: No contrast discernible. No DWI/ADC changes

- $\quad$ Score 1: Poor contrast with $<20 \%$ correlation with NPV post contrast

- $\quad$ Score 2: Below average contrast with 20-50\% correlation with post-contrast NPV

- $\quad$ Score 3: Average contrast with 50\% correlation with post-contrast NPV

- Score 4: Good contrast with 50-80\% correlation with post-contrast NPV

- Score 5: Excellent contrast and $>80 \%$ correlation with post-contrast NPV

The NPVs were assessed from the cT1WIs, DWIs and ADC maps by two independent senior radiologists using the inherent volume calculation software in the MRI workstation (Signa HDxt 1.5T, General Electric Healthcare, USA). The radiologists independently and manually contoured the non-enhanced / devascularized regions by summation of sequential axial slices on the different MR images to calculate the NPV. All evaluation and measurement were performed under a blinded condition to avoid bias. The NPV ratios of the fibroid and adenomyoma were calculated by dividing the NPV with the lesion volume measured on the T2WI before the treatment.

\section{Statistical analysis}

The reproducibility of the image contrast scores and NPV values obtained from two independent radiologists were examined using interclass correlation coefficient
(ICC) where a coefficient of above 0.90 was considered as 'good' reliability. The image contrast scores, NPVs and NPV ratios as measured from CT1WIs well as from DWIs and ADC maps of different $b$-values, were compared in pairs using Wilcoxon signed-rank test, whereas the differences among the image contrast scores, NPVs and NPV ratios as measured on DWIs or ADC maps, were analyzed using Kruskal-Wallis test. A confidence interval of $95 \%$ was used and the difference was considered as statistically significant at $p<0.05$. In addition, the linear regression analysis was performed and the correlation coefficient was calculated for the NPVs obtained from cT1WIs as well as from DWIs and ADC maps of different $b$-values. The statistical analysis was performed using SPSS statistical software (version 22.0, IBM, New York, USA).

\section{Results}

\section{Patient characteristics}

Nine fibroid and five adenomyoma patients (39 \pm 8 years old), with no concurrent medical disease or previous surgery, have participated in the study and all patients underwent MRgFUS treatment successfully. No adverse reaction was reported except that one patient experienced a mild degree of superficial abdominal skin burn immediately after treatment. Following MRgFUS treatment, eleven patients managed to be followed up after 12 months while two fibroid patients were lost in the follow-up and one adenomyoma patient had undergone a hysterectomy due to persistent menorrhagia. The flow of patients' recruitment in the study is presented in Figure 1.

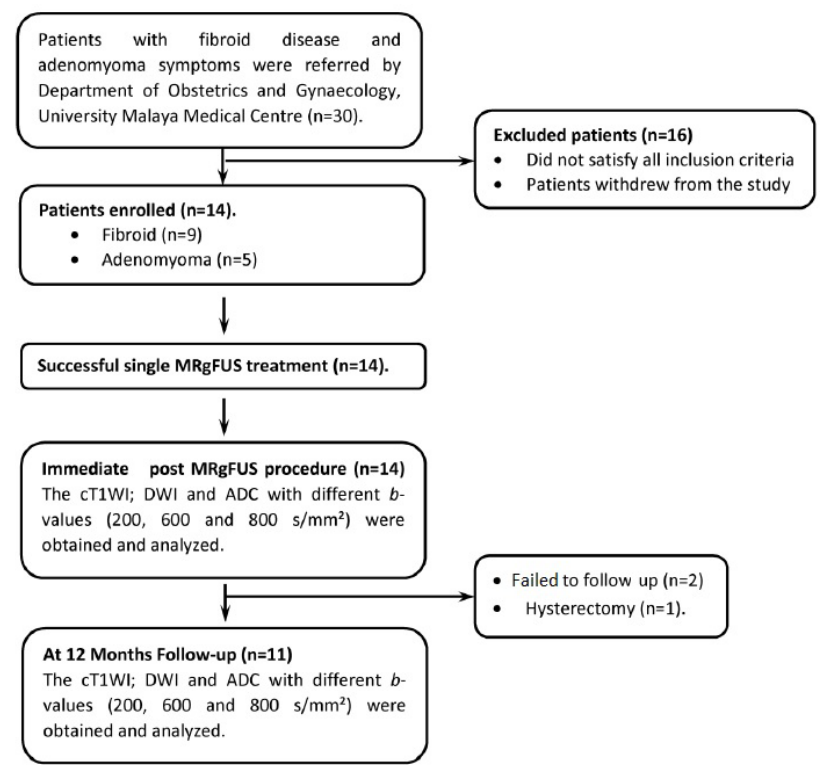

Figure 1: Flow chart of patient's recruitment in the study

\section{Lesion characteristics}

Signal intensity changes were not noted in any of the cT1WIs, DWIs and ADC maps acquired before the treatment, indicating no necrosis or degeneration within the lesion. Immediately after the treatment, the ablated regions on DWIs demonstrated hyperintense signals, showing a good 
visual agreement with the hypointense non-perfused regions on the CT1WIs. The signal intensity changes on ADC maps (mainly hypointense signals) appeared to be inconsistent and varied between different patients where some lesions showed a mixed and heterogeneous signal intensity change. At 12 months follow-up, despite some changes in size and appearance, the non-perfused regions were found persistent on the cT1WIs of all patients. The signal intensity of the ablated regions on DWIs and ADC maps appeared to be heterogeneous in some cases (mainly on ADC maps). Figures 2 and 3 showed the examples of MR images acquired during pre-treatment, immediately after treatment and at 12 months follow-up, for fibroid and adenomyoma patients, respectively.

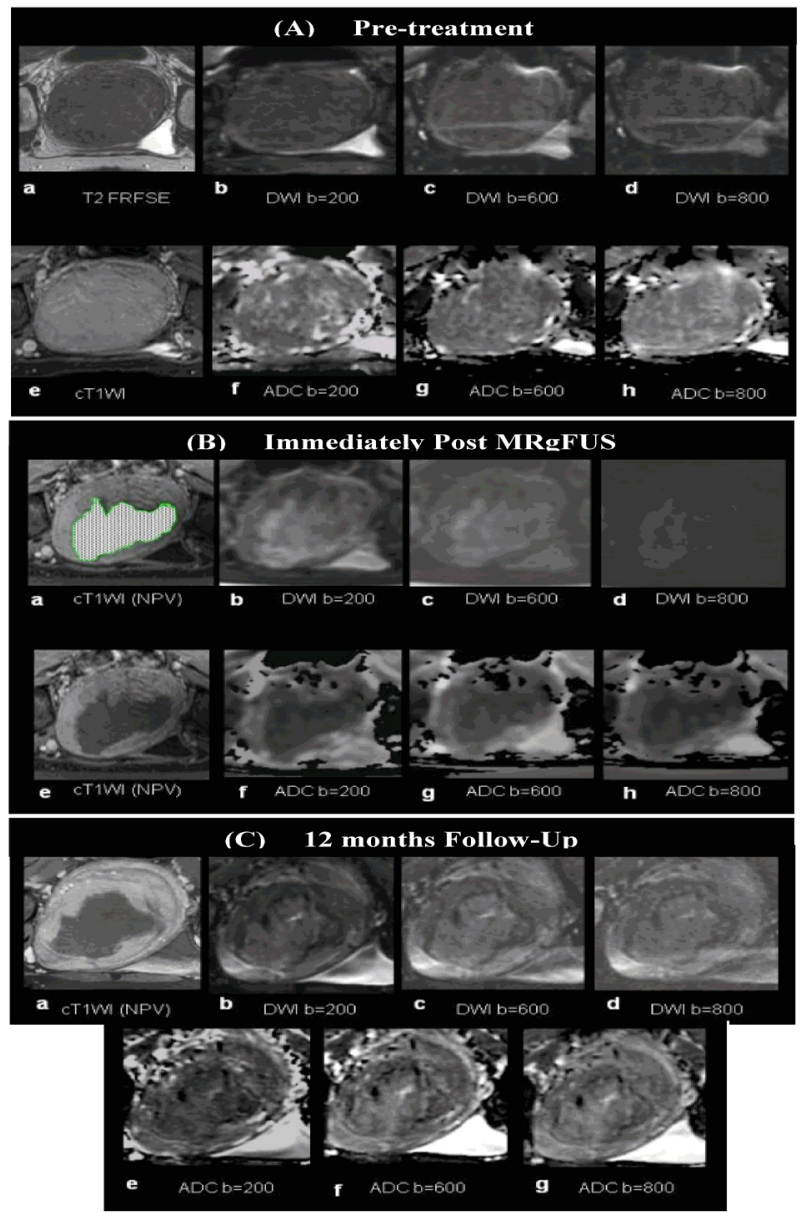

Figure 2: Typical T2WI, cT1WI, DWIs and ADC maps $\left(b\right.$-values $=200,600$ and $\left.800 \mathrm{~s} / \mathrm{mm}^{2}\right)$ of uterine fibroid patient. (A) Before MRgFUS treatment: Pre-treatment MRI showed a hypointense uterine fibroid on axial T2WI which enhanced homogenously on CT1WI. No areas of restricted diffusion observed on DWI or ADC maps of different $b$-values before MRgFUS. (B) Immediately after the MRgFUS treatment: Non-perfused region was observed on cT1WI, DWIs and ADC maps. DWI of $200 \mathrm{~s} / \mathrm{mm}^{2}$ showed a clearer non-perfused area compared to other DWIs and all ADC maps. (C) At 12 months follow-up: Non-perfused area on CT1WI persisted. Heterogeneous area was observed on all DWIs and ADC maps. The DWIs of different $b$-values showed a clearer delineation area than ADC maps

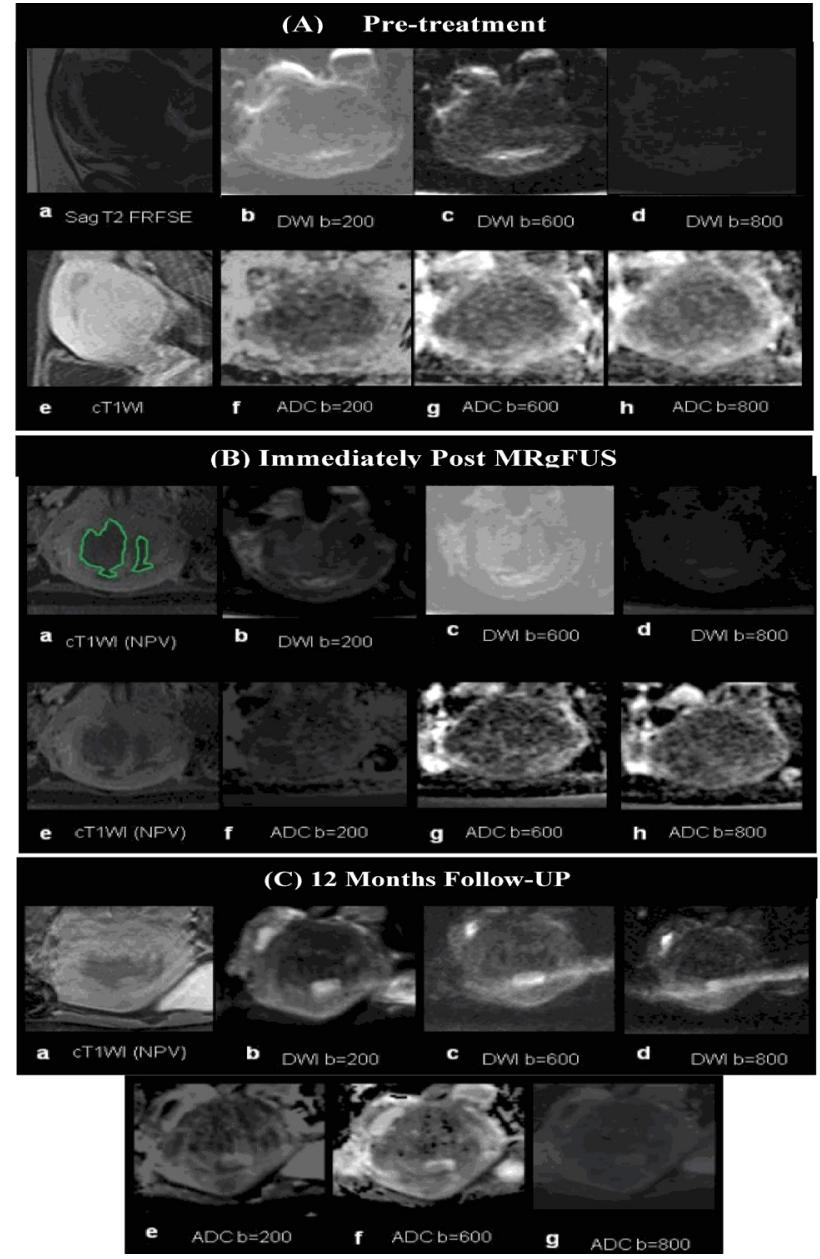

Figure 3: Typical T2WI, cT1WI, DWIs and ADC maps $\left(b\right.$-values $=200,600$ and $\left.800 \mathrm{~s} / \mathrm{mm}^{2}\right)$ of adenomyoma patient. (A) Before MRgFUS treatment: Sagittal T2WI showed focal adenomyosis with contrast enhancement on cT1WI. No areas of restricted diffusion seen on all DWIs and ADC maps of different $b$-values. (B) Immediately after the MRgFUS treatment: Non-perfused region was observed on cT1WI. Hyperintense area observed on DWIs while ADC maps showed no significant intensity changes. Clearer nonperfused area seen on DWI of $200 \mathrm{~s} / \mathrm{mm}^{2}$ compared to other DWIs and all ADC maps. (C) At 12 months follow-up: Nonperfused area on CT1WI persisted. Non-perfused area was appreciated on the DWIs. A poor delineation of nonperfused area and poor contrast between ablated and non-ablated regions were observed on ADC maps due to low signal to noise ratio

\section{Image contrast score}

A good inter-observer agreement (ICC $=0.992$ ) were observed between the image contrast scores obtained from two independent radiologists and therefore an average reading was used in subsequent analysis. The image contrast scores of cT1WIs, DWIs and ADC maps of patients can be visualized from Figure 4 and the scores are listed in Supplementary Table 1. The cT1WI has demonstrated the best contrast between the ablated and non-ablated regions, and therefore was given a full score of 5 . 
The contrast scores of DWIs and ADC maps of different $b$-values obtained immediately after the treatment were significantly lower $(p<0.05)$ than the contrast score of cT1WIs (Figure 4A and Table 1 ). Significantly higher contrast scores $(p<0.05)$ were obtained from the DWIs compared to the ADC maps of the same $b$-values. Among the DWIs of different $b$-values, the DWI obtained with the lowest $b$-values of $200 \mathrm{~s} / \mathrm{mm}^{2}$ has demonstrated the best contrast. However, the contrast scores obtained for DWIs of different $b$-values did not vary significantly ( $p=0.238$ ) from each other. The same observation was found for the ADC maps of different $b$-value where the $\mathrm{p}$ value of 0.989 was obtained from the Kruskal-Wallis test.

At 12 months follow-up, the image contrast scores of all DWIs and ADC maps were essentially lower $(p<0.05)$ than cT1WIs (Figure 4B). Although ADC maps did produce a poorer contrast compared to DWIs, however, the score difference between ADC maps and DWIs of different $b$-values were found not to be significant $(p>0.05)$. The contrast scores of DWIs of different $b$-values were also found not significantly different $(p=0.823)$ despite showing a generally better scores for DWIs of higher $b$-values (600 and $\left.800 \mathrm{~s} / \mathrm{mm}^{2}\right)$. A minimal score difference between the ADC maps of different $b$-values $(p=0.885)$ were also observed.

A

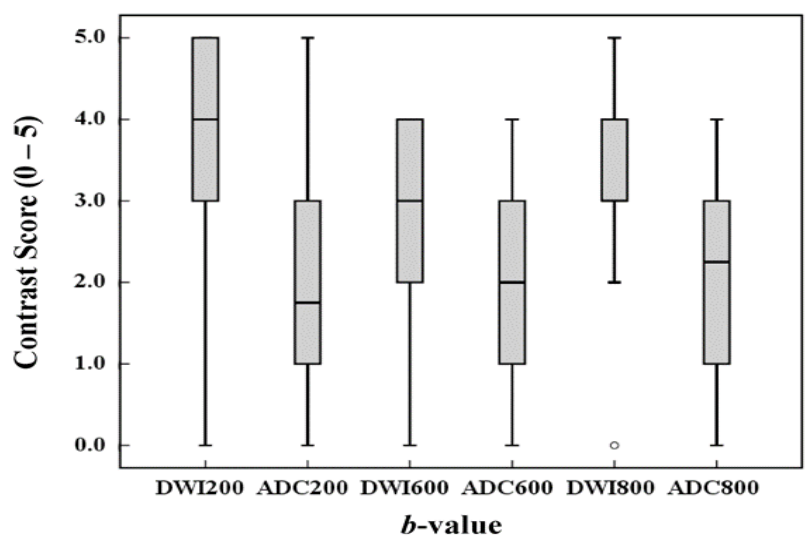

B

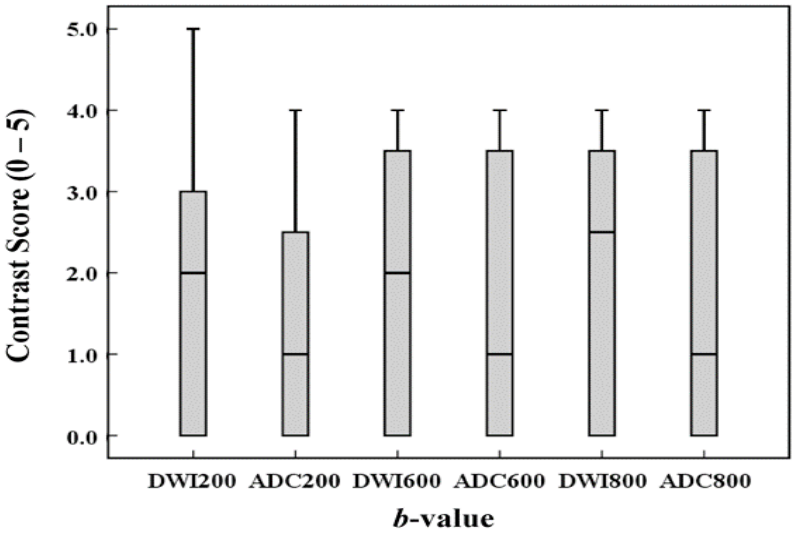

Figure 4: Image contrast score obtained for DWIs and ADC maps, immediately after (A) and 12 months after (B) MRgFUS treatment
Table 1: Statistical comparison of contrast scores of cT1WI as well as DWIs and ADC maps of different $b$-values, immediately after MRgFUS treatment and at 12 months follow-up

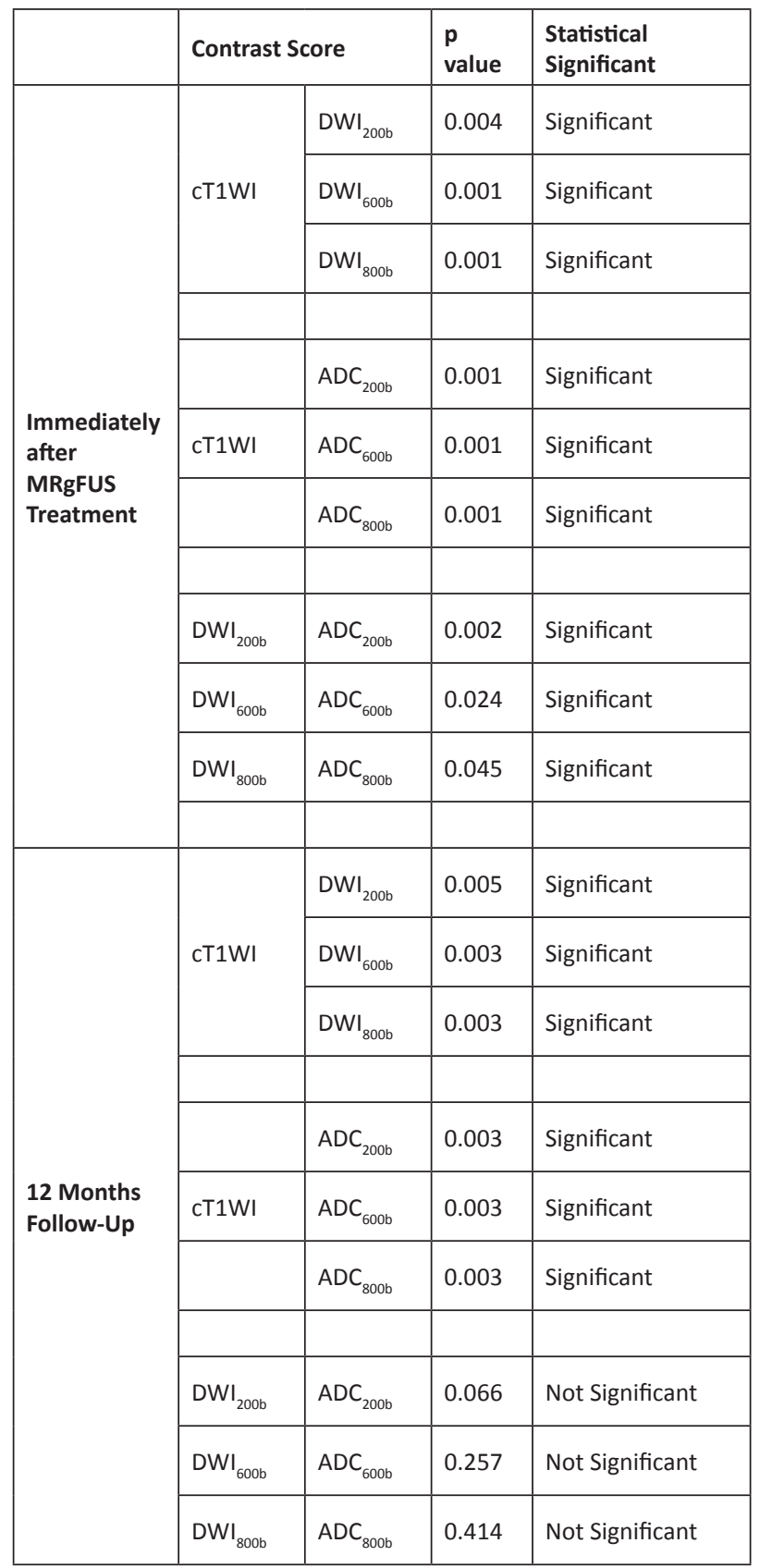

\section{Quantitative data analysis}

An inter-class coefficient of 1.00 has been obtained for the NPVs measured by two independent radiologists and hence the average NPV values were used in subsequent analysis. The NPVs of all patients measured on CT1WIs as well as on DWIs and ADC maps of different $b$-values, immediately after, and at 12 months after the treatment, are listed in Table 2. While the NPVs obtained from ADC maps of different $b$-values as well as from DWIs of 600 and $800 \mathrm{~s} / \mathrm{mm}^{2}$ were significantly different from the postcontrast NPV $(p<0.05)$ immediately after treatment, only the NPV obtained from DWI of $200 \mathrm{~s} / \mathrm{mm}^{2}$ were similar to the post-contrast NPV ( $p=0.059)$. The NPVs obtained 
from DWIs were also significantly different from the NPVs obtained from ADC maps at $p<0.05$ (Table 3). At 12 months follow-up, the NPVs obtained from DWIs and ADC maps of different $b$-values were found similar to the post-contrast NPV ( $p>0.05)$. The NPVs of DWIs and NPVs of ADC maps of the same $b$-value were also not significantly different ( $p>0.05$ ), except those obtained with $b$-value of $200 \mathrm{~s} /$ $\mathrm{mm}^{2}$ (Table 3).

The linear regression analysis was performed on the NPVs obtained from DWIs, ADC maps and CT1WIs, both immediately after the treatment (Figure $5 \mathrm{~A}-\mathrm{C}$ ), and at 12 months follow-up (Figure 5D-F). The NPVs obtained from DWIs of different $b$-values immediately after the treatment were found highly correlated with the post-contrast NPV as the correlation factors were all greater than 0.80. The NPV obtained from DWI of $200 \mathrm{~s} / \mathrm{mm}^{2}$ showed the strongest correlation to the post-contrast NPV at $\mathrm{R}^{2}=0.983$. In comparison, the correlation between post-contrast NPV and NPVs of different ADC maps were found weaker (53-59\%) immediately after the treatment (Figure 5A-C). The correlation between DWIs and ADC maps were found stronger (59-78\%) than the correlation between NPVs of ADC maps and CT1WIs, with the strongest correlation found between $\mathrm{DWI}_{800 \mathrm{~b}}$ and $\mathrm{ADC}_{800 \mathrm{~b}}\left(R^{2}=0.783\right)$.

12 months follow-up, a good correlation (73-95\%) was observed between the post-contrast NPV and the NPVs of DWIs and ADC maps of different $b$-values (Figure $5 D-F)$. The NPVs of $D^{2} I_{200 b}$ and $A D C_{200 b}$ map showed approximate $84 \%$ correlation with the post-contrast NPV, whereas slightly weaker correlations were observed for NPVs of DWIs and ADC maps of 600 and $800 \mathrm{~s} / \mathrm{mm}^{2}$. The correlation between the NPVs of DWIs and ADC maps of different $b$-values showed the strongest correlation ( $98 \%$ ) observed at 12 months follow-up (Figure 5D-F).
A

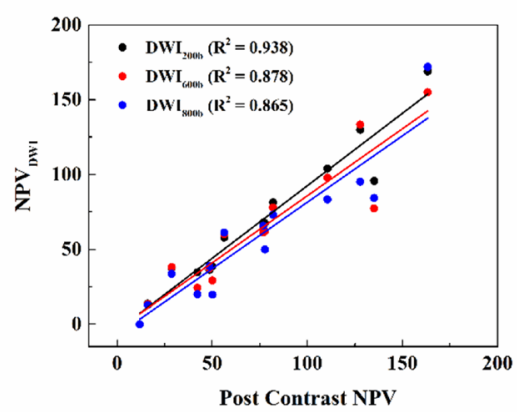

D

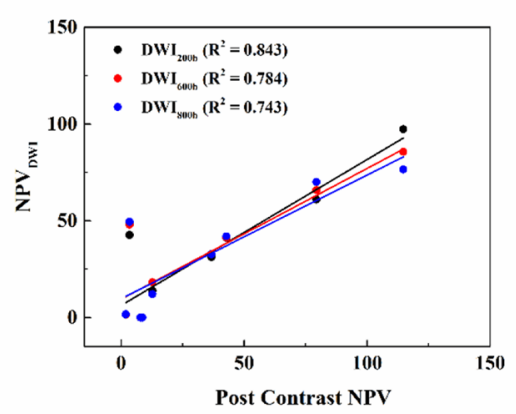

B

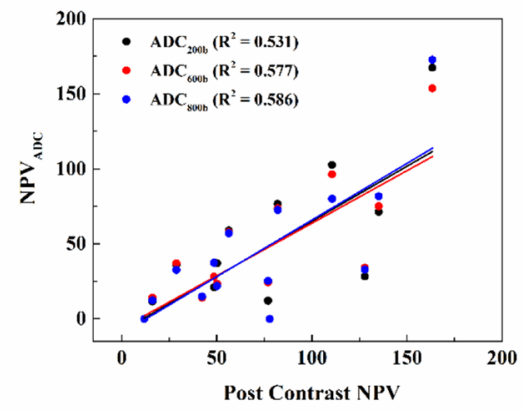

$\mathbf{E}$

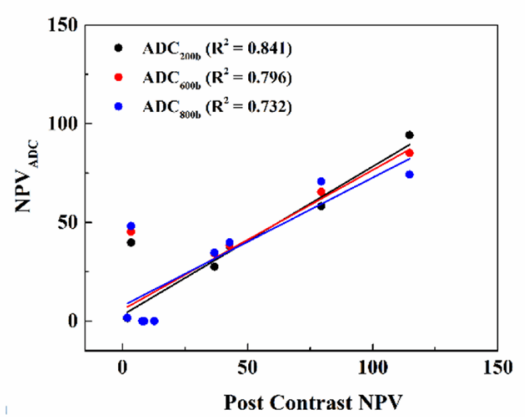

C

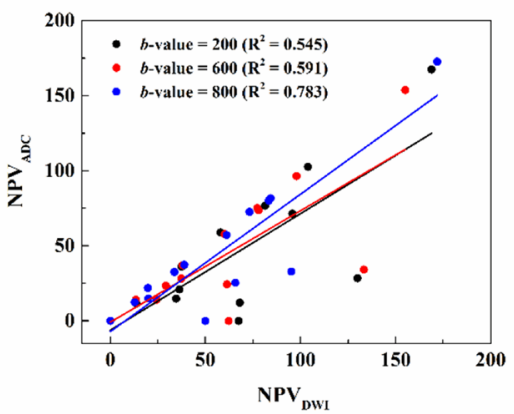

F

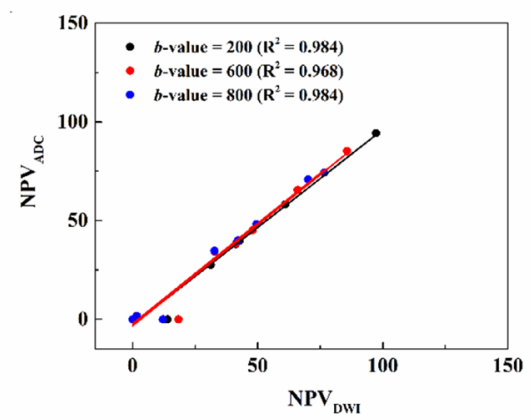

Figure 5: Linear regression analysis of the NPVs obtained from cT1WI, DWIs and ADC maps, immediately after (A-C), and 12 months after (D-F), MRgFUS treatment 


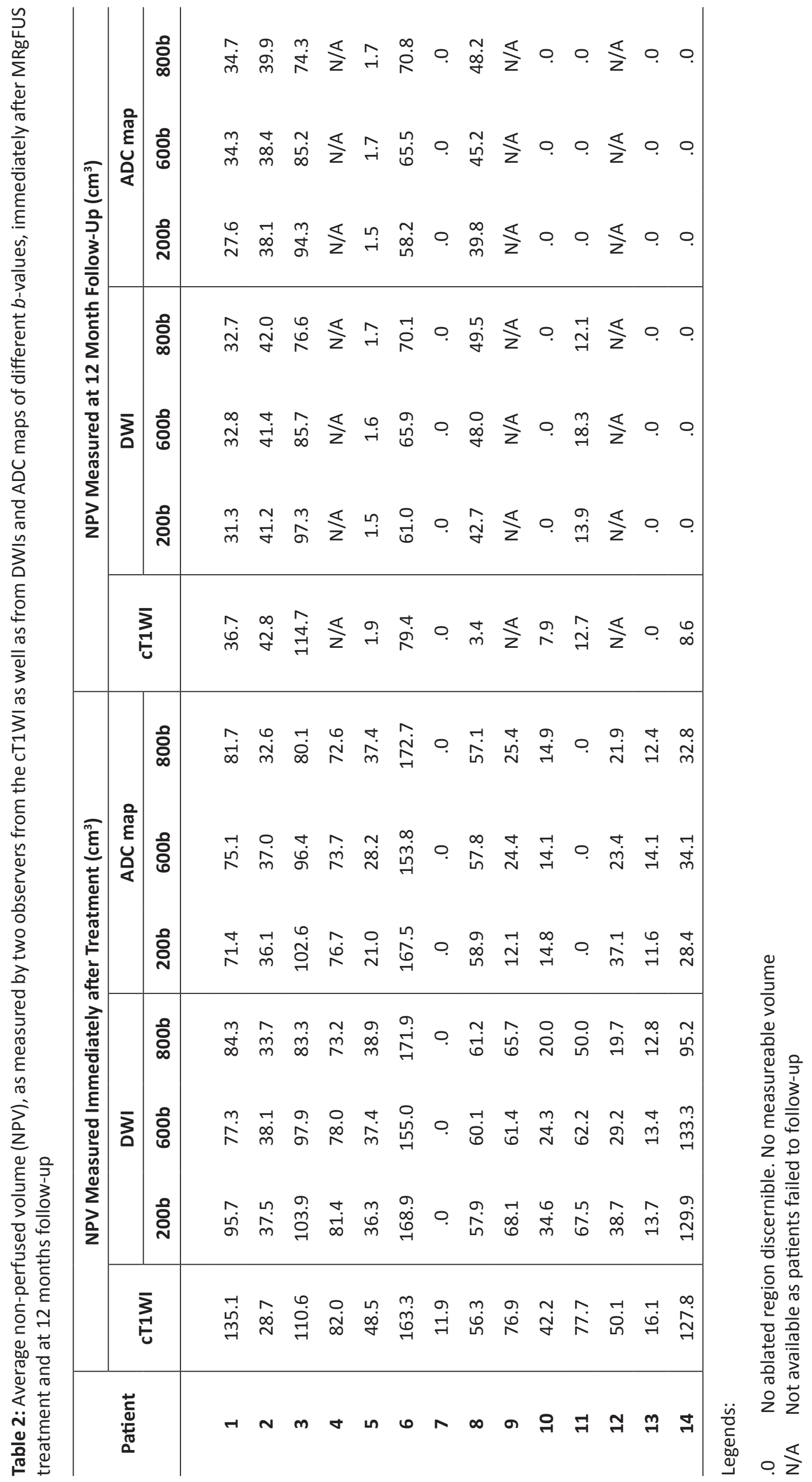


Table 3: Statistical comparison of non-perfused volume (NPV), as calculated from cT1WI, as well as from DWIS and ADC maps of different $b$-values, immediately after MRgFUS treatment and at 12 months follow-up

\begin{tabular}{|c|c|c|c|c|}
\hline & \multicolumn{2}{|c|}{ Contrast Score } & \multirow{2}{*}{$\begin{array}{l}\text { p value } \\
0.059\end{array}$} & \multirow{2}{*}{$\begin{array}{l}\text { Statistical Significant } \\
\text { Not significant }\end{array}$} \\
\hline & & $\mathrm{DWI}_{200 b}$ & & \\
\hline & cT1WI & $D W I_{600 b}$ & 0.019 & Significant \\
\hline & & $\mathrm{DWI}_{800 \mathrm{~b}}$ & 0.011 & Significant \\
\hline & & $A D C_{200 b}$ & 0.009 & Significant \\
\hline \multirow{9}{*}{$\begin{array}{l}\text { Immediately after MRgFUS } \\
\text { Treatment }\end{array}$} & cT1WI & $\mathrm{ADC}_{600 \mathrm{~b}}$ & 0.004 & Significant \\
\hline & & $A D C_{800 b}$ & 0.010 & Significant \\
\hline & $\mathrm{DWI}_{200 \mathrm{~b}}$ & $A D C_{200 b}$ & 0.002 & Significant \\
\hline & $\mathrm{DWI}_{600 \mathrm{~b}}$ & $\mathrm{ADC}_{600 \mathrm{~b}}$ & 0.002 & Significant \\
\hline & $\mathrm{DWI}_{800 \mathrm{~b}}$ & $A D C_{800 b}$ & 0.011 & Significant \\
\hline & & $\mathrm{DWI}_{200 \mathrm{~b}}$ & 0.398 & Not significant \\
\hline & cT1WI & $\mathrm{DWI}_{600 \mathrm{~b}}$ & 0.612 & Not significant \\
\hline & & $\mathrm{DWI}_{800 \mathrm{~b}}$ & 0.237 & Not significant \\
\hline & & $A D C_{200 b}$ & 0.237 & Not significant \\
\hline \multirow[t]{5}{*}{12 Months Follow-Up } & cT1WI & $\mathrm{ADC}_{600 \mathrm{~b}}$ & 0.237 & Not significant \\
\hline & & $\mathrm{ADC}_{800 \mathrm{~b}}$ & 0.237 & Not significant \\
\hline & $\mathrm{DWI}_{200 \mathrm{~b}}$ & $\mathrm{ADC}_{200 \mathrm{~b}}$ & 0.028 & Significant \\
\hline & $\mathrm{DWI}_{600 \mathrm{~b}}$ & $\mathrm{ADC}_{600 \mathrm{~b}}$ & 0.128 & Not significant \\
\hline & $D W I_{800 b}$ & $A D C_{800 b}$ & 0.173 & Not significant \\
\hline
\end{tabular}

\section{Therapeutic outcome analysis}

The NPV ratio served as a reflection of the ablation percentage where the ablation percentage is equal to NPV ratio of $100 \%$. The NPV ratios were calculated for all treated lesions immediately after the treatment and at 12 months follow-up (Table 4). The statistical analysis results of NPV ratio obtained from cT1WI, DWIs and ADC maps are given in Table 5. Immediately after the treatment, only the NPV ratio derived from DWI of $200 \mathrm{~s} / \mathrm{mm}^{2}$ was essentially similar to the post-contrast NPV ratio $(p=0.075)$, while the NPV ratios of other DWIs and all ADC maps were statistically different from the post-contrast NPV ratio $(p<0.05)$. The
NPV ratios obtained from DWIs were also different from the NPV ratios obtained from ADC maps at $p<0.05$ (Table 5).

At 12 months follow-up, the NPV ratio derived from cT1WI was found similar to the NPV ratios obtained from all DWIs and ADC maps of different $b$-values $(p>0.05)$. It should be noted from the Table 5, only the differences between the NPV ratios of DWI and ADC map of $200 \mathrm{~s} / \mathrm{mm}^{2}$ were considered significant, while the NPV ratios of DWIs and ADC maps of $600 \mathrm{~s} / \mathrm{mm}^{2}$ and $800 \mathrm{~s} / \mathrm{mm}^{2}$ were similar to each other. 


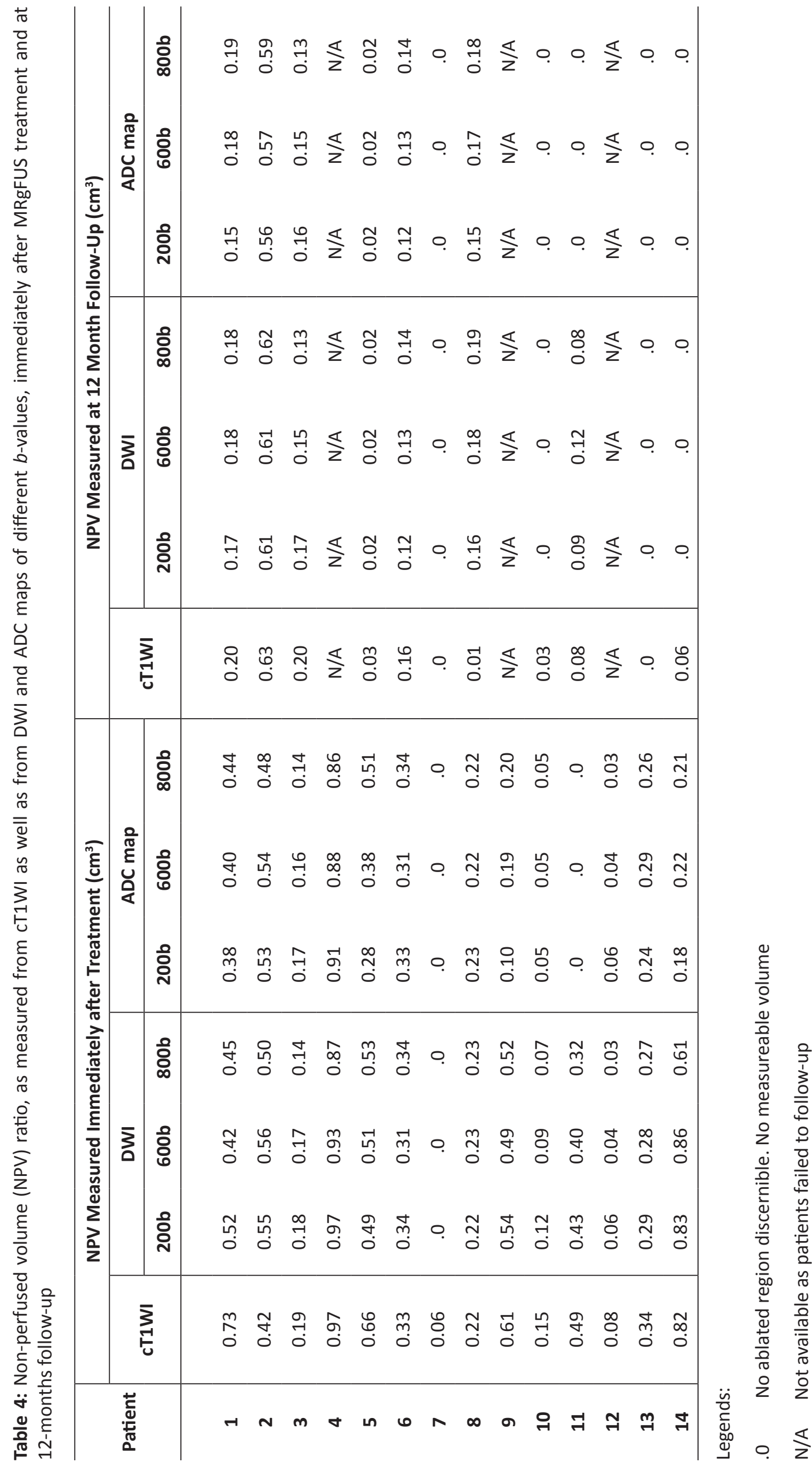


Table 5: Statistical comparison of non-perfused volume (NPV) ratio, as calculated from cT1WI as well as from DWIs and ADC maps of different $b$-values, immediately after MRgFUS and at 12 months follow-up

\begin{tabular}{|c|c|c|c|c|}
\hline & Contras & core & $\begin{array}{l}p \\
\text { value }\end{array}$ & $\begin{array}{l}\text { Statistical } \\
\text { Significant }\end{array}$ \\
\hline \multirow{13}{*}{$\begin{array}{l}\text { Immediately } \\
\text { after MRgFUS } \\
\text { Treatment }\end{array}$} & \multirow{3}{*}{ cT1WI } & $\mathrm{DWI}_{200 \mathrm{~b}}$ & 0.059 & $\begin{array}{l}\text { Not } \\
\text { significant }\end{array}$ \\
\hline & & $\mathrm{DWI}_{600 \mathrm{~b}}$ & 0.019 & Significant \\
\hline & & $\mathrm{DWI}_{800 \mathrm{~b}}$ & 0.011 & Significant \\
\hline & \multirow{3}{*}{ cT1WI } & $\mathrm{ADC}_{200 b}$ & 0.009 & Significant \\
\hline & & $A D C_{600 b}$ & 0.004 & Significant \\
\hline & & $A D C_{800 b}$ & 0.010 & Significant \\
\hline & $\mathrm{DWI}_{200 \mathrm{~b}}$ & $\mathrm{ADC}_{200 \mathrm{~b}}$ & 0.002 & Significant \\
\hline & $\mathrm{DWI}_{600 \mathrm{~b}}$ & $\mathrm{ADC}_{600 \mathrm{~b}}$ & 0.002 & Significant \\
\hline & $\mathrm{DWI}_{800 \mathrm{~b}}$ & $A D C_{800 b}$ & 0.011 & Significant \\
\hline & & $\mathrm{DWI}_{200 \mathrm{~b}}$ & 0.398 & $\begin{array}{l}\text { Not } \\
\text { significant }\end{array}$ \\
\hline & cT1WI & $\mathrm{DWI}_{600 \mathrm{~b}}$ & 0.612 & $\begin{array}{l}\text { Not } \\
\text { significant }\end{array}$ \\
\hline & & $\mathrm{DWI}_{800 \mathrm{~b}}$ & 0.237 & $\begin{array}{l}\text { Not } \\
\text { significant }\end{array}$ \\
\hline & \multirow{3}{*}{ cT1WI } & $A D C_{200 b}$ & 0.237 & $\begin{array}{l}\text { Not } \\
\text { significant }\end{array}$ \\
\hline \multirow[t]{5}{*}{$\begin{array}{l}12 \text { Months } \\
\text { Follow-Up }\end{array}$} & & $A D C_{600 b}$ & 0.237 & $\begin{array}{l}\text { Not } \\
\text { significant }\end{array}$ \\
\hline & & $\mathrm{ADC}_{800 \mathrm{~b}}$ & 0.237 & $\begin{array}{l}\text { Not } \\
\text { significant }\end{array}$ \\
\hline & $\mathrm{DWI}_{200 \mathrm{~b}}$ & $A D C_{200 b}$ & 0.028 & Significant \\
\hline & $\mathrm{DWI}_{600 \mathrm{~b}}$ & $A D C_{600 b}$ & 0.128 & $\begin{array}{l}\text { Not } \\
\text { significant }\end{array}$ \\
\hline & $\mathrm{DWI}_{800 \mathrm{~b}}$ & $A D C_{800 b}$ & 0.173 & $\begin{array}{l}\text { Not } \\
\text { significant }\end{array}$ \\
\hline
\end{tabular}

\section{Discussion}

The cT1WI is the gold standard for the assessment of a region or volume after MRgFUS treatment. Due to the relatively lower contrast uptake by the ablated region, it appeared as a hypointense region on CT1WI, and is known as a non-perfused region. The volume of the non-perfused region has been widely used for treatment effectiveness measurement. On the other hand, the ablated regions on the DWIs and ADC maps were formed due to, either the perfusion effect from vessel destruction during the treatment, or cytotoxic oedema. The DWI and ADC maps can be used to evaluate the peri- and post-procedural treatment outcome for MRgFUS treatment of uterine fibroid and adenomyoma. The peri-procedural monitoring using DWI and ADC maps could provide more information and feedback to the physician on the treatment planning and tumour ablation. For the post-procedural treatment outcome evaluation, the non-perfused region can be delineated from both the DWI and ADC maps for the determination of NPV and NPV ratio.

The DWI of $200 \mathrm{~s} / \mathrm{mm}^{2}$ has demonstrated the best image contrast and displayed the clearest visualization of the ablation border compared to other DWIs and ADC maps immediately after the treatment. This observation was in agreement with previously published data, suggesting the lower $b$-value would have produced a better image contrast $(17,23)$. The poorer image contrast of DWls obtained with $b$-values of $600 \mathrm{~s} / \mathrm{mm}^{2}$ and $800 \mathrm{~s} / \mathrm{mm}^{2}$ can be ascribed to low signal-to-noise ratio due to shorter $\mathrm{T} 2$ relaxation times (25). Immediately after the treatment, the appearance of the treated regions on all the ADC maps appeared to be unpredictable, similar to those observed by Pilatou et al. (22). The treated regions on ADC maps showed significant areas of heterogeneity which perhaps reflected the differences in the tissue cellularity and properties. The poor contrast resolution of ADC maps obtained at lower $b$-values was also noticed as the result of low signal attenuation. However, a slight improvement in image resolution was appreciated in the ADC map of $800 \mathrm{~s} / \mathrm{mm}^{2}$ as a higher signal attenuation was achieved at the higher $b$-value.

At 12 months follow-up, the image contrast of both DWIs and ADC maps of different $b$-values were generally poor due to heterogeneity of the ablated region and hence yielded a lower image contrast score. Since no significant difference was noticed between the contrast scores of DWIs and ADC maps of different $b$-values, there was no specified $b$-value to be considered as superior in delineating the ablated region at 12 months follow-up. Our results appear to contradict earlier reports $(17,23)$ that suggested a better ADC map contrast was produced with higher $b$-values (400, 600 and $800 \mathrm{~s} / \mathrm{mm}^{2}$ ) during 1 month follow-up due to the diffusion effects. Such differences noted can be ascribed to the different length of the follow-up period ( 1 month in the earlier reports against 12 months in our study). In view of this, a further investigation on a larger scale should be performed to determine the influence of different $b$-values on image contrast at different follow-up periods.

The NPVs as measured from the DWIs and ADC maps showed an under-estimated ablation volume in most of the patients when compared to the post-contrast NPV, both immediately after and 12 months after the treatment. The NPV derived from DWIs showed a better correlation to the post-contrast NPV compared to those obtained from ADC maps with the best correlation demonstrated by the DWI of $200 \mathrm{~s} / \mathrm{mm}^{2}$. The higher image contrast of the DWI obtained with $b$-value of $200 \mathrm{~s} / \mathrm{mm}^{2}$ has allowed a better delineation 
and a higher measurement accuracy of the ablated region. This finding was in agreement with the results reported earlier $(17,23)$, hence suggesting a better depiction of the ablated region on the lower $b$-value images. Furthermore, it has been suggested that the visual comparison of the DWIs of higher $b$-values to the contrast-enhanced MR images are less precise $(17,23)$. The NPVs derived from all $A D C$ maps have demonstrated a poor correlation with the post-contrast NPV, which can be attributable to the poorer image contrast of the ADC maps. The low signal-to-noise ratio and signal heterogeneity seemed to be responsible for the poorer image contrast of ADC maps.

At 12 month follow-up, a notable decrease in the NPVs was observed on all the patients accessed. Both the DWIs and the ADC maps showed an inconsistent and more heterogeneous signal changes at 12 months follow-up. There were four patients who showed no measurable NPVs on the DWIs and ADC maps. Out of these four patients, two of them had also no measurable post-contrast NPV. The patients who demonstrated no discernible DWI or ADC map signal changes at follow-up were the adenomyoma patients where three of the adenomyoma patients did not show any signal changes on both DWIs and ADC maps while the remaining patient, who although demonstrated DWI signal changes, did not show apparent ADC map signal changes. The main reason for the above observation was not fully understood but it may be due to the nature of the lesion where the tissue recovery rate and response to the MRgFUS procedure might vary. Anyhow, these observations indicated the limitation of using DWI or ADC map to assess the treatment outcome of MRgFUS during followup periods. Except for those cases mentioned above, the delineation of the NPV was still possible in the remaining cases at 12 months follow-up. There was a good correlation with no significant difference between the post-contrast NPV and volumes derived from all the DWIs and ADC maps of different $b$-values at follow-up.

The NPV ratio calculated from cT1WI, DWIs and ADC maps reflected the percentage of ablation. Immediately after the treatment, the NPV ratios calculated from the DWI of $200 \mathrm{~s} / \mathrm{mm}^{2}$ correlated well with the post-contrast NPV ratio, suggesting the DWI with a lower $b$-value could be employed as a measurement parameter to assess the MRgFUS treatment outcome. At 12 months follow-up, the NPV ratios calculated from DWIs and ADC maps of different $b$-values correlated well with the post-contrast NPV ratio. The smaller lesion size, and hence, lower NPV ratio at follow-up has allowed a lesser error in ablated region delineation and this may be responsible for the above observation.

There are several advantages for the application of DWI and ADC maps for MRgFUS treatment outcome evaluation. Despite being used for post-procedural outcome evaluation, the DWI and ADC maps can be used for peri-procedural monitoring as no contrast agent is involved. In addition, the ablation can be performed immediately after the post-procedural MR scanning for the patient with incomplete tumour ablation, as hazardous potential of heating the contrast agent is not involved for DWI and ADC maps. The DWI and ADC maps also enabled the non-invasive evaluation of multiple ablated lesions simultaneously and provided a faster imaging time. In addition, the DWI and ADC maps obviate the contrast agent injection which decreases the possible chance of systemic reaction to the contrast agent. As the method does not involve the use of contrast agent, the treatment cost may be further reduced (18). Nonetheless, the DWI and ADC maps are limited by the motion artefacts. Although use of echo-planar MR imaging for a patient lying in a prone position with conscious sedation may reduce the motion artefacts, it may be a major limitation, for the use of DWI and ADC maps. Furthermore, some patients may show a poorer contrast in the DWI and ADC maps which may lead to inaccurate delineation of the non-perfused area and thus the NPV calculation. Hence, selection of the $b$-value that gives a better contrast of DWI and ADC maps is crucial.

A major limitation of this study was the small sample size as only a limited number of patients fulfilled the inclusion criteria, and some patients were lost during follow-up. Since this was a preliminary study involving the use of multiple $b$-values for DWI and ADC maps, a larger sample size study, preferably, a randomized controlled trial from multi-centres, is needed to assess the efficacy of DWIs and ADC maps of different $b$-values to evaluate the treatment outcome of MRgFUS of uterine fibroids and adenomyoma.

\section{Conclusion}

In conclusion, this study demonstrated the feasibility and effectiveness of treated uterine fibroid or adenomyoma volume measurements after MRgFUS treatment using DWIs and ADC maps of different $b$-values. The DWI obtained with a low $b$-value $\left(200 \mathrm{~s} / \mathrm{mm}^{2}\right)$ is a feasible alternative for delineation and quantitative volumetric evaluation of ablated region immediately after the treatment. Despite some degree of under-estimation, the ablated volume measured from the DWI of $200 \mathrm{~s} / \mathrm{mm}^{2}$ was comparable to the volume obtained from cT1WI. Furthermore, our study also demonstrated a good image contrast and showed a good correlation with the post-contrast NPV ratio. The DWI obtained with a higher $b$-value $\left(600\right.$ and $800 \mathrm{~s} / \mathrm{mm}^{2}$ ) was found less accurate for volume measurement while all the ADC maps of different $b$-value showed a poor image contrast and significant NPV under-estimation.

At 12 months follow-up, all the DWIs and ADC maps of different $b$-values are feasible for ablated volume calculations. However, the DWI and ADC maps obtained with a lower $b$-value of $200 \mathrm{~s} / \mathrm{mm}^{2}$ remained superior compared to the images obtained with higher $b$-values in terms of volume correlation with post-contrast NPV. However, since there were a few patients who demonstrated no discernible DWI / ADC map signal changes at follow up, the role of DWI as a reliable surrogate for contrast-enhanced imaging, after MRgFUS treatment to measure the treated volume, remains questionable and warrants a further larger scale study. Nevertheless, DWI 
remains a useful method to evaluate the regions that have been thermally ablated by MRgFUS.

\section{Conflict of interests}

The authors declare no conflict of interests.

\section{Acknowledgements}

The authors thank the Department of Biomedical Imaging, University of Malaya and University of Malaya Medical Centre, for providing necessary facilities to carry out this work.

\section{References}

1. Cramer SF, Patel A. The frequency of uterine leiomyomas. Am J Clin Pathol. 1990; 94(4):435-8.

2. Stewart EA. Uterine fibroids. Lancet. 2001; 357(9252):293-8.

3. Carlson KJ, Nichols DH, Schiff I. Indications for hysterectomy. N Engl J Med. 1993; 328(12):856-60.

4. Parker WH. Uterine myomas: management. Fertil Steril. 2007; 88(2):255-71.

5. Pron G, Bennett J, Common A, Wall J, Asch M, Sniderman K. The Ontario uterine fibroid embolization trial. Part 2. Uterine fibroid reduction and symptom relief after uterine artery embolization for fibroids. Fertil Steril. 2003; 79(1):120-7.

6. Gynecologists ACoOa. Surgical alternatives to hysterectomy in the management of leiomyomas acog practice bulletin \#16. Int J Gynaecol Obstet. 2000:285-94

7. Dong $X$, Yang Z. High-intensity focused ultrasound ablation of uterine localized adenomyosis. Curr Opin Obstet Gynecol. 2010; 22(4):326-30.

8. Fukunishi H, Funaki K, Sawada K, Yamaguchi K, Maeda T, Kaji Y. Early results of magnetic resonanceguided focused ultrasound surgery of adenomyosis: Analysis of 20 cases. J Minim Invasive Gynecol. 2008; 15(5):571-9.

9. Gedroyc WM. MR-guided focused ultrasound treatment of uterine fibroids. In: Kahn T, Busse $\mathrm{H}$, editors. Interventional Magnetic Resonance Imaging. Berlin, Heidelberg: Springer Berlin Heidelberg. 2012:341-8.

10. Hesley GK, Felmlee JP, Gebhart JB, Dunagan KT, Gorny $\mathrm{KR}$, Kesler JB, et al. Noninvasive treatment of uterine fibroids: early Mayo clinic experience with magnetic resonance imaging-guided focused ultrasound. Mayo Clin Proc. 2006; 81(7):936-42.

11. Morita Y, Ito N, Hikida H, Takeuchi S, Nakamura K, Ohashi $\mathrm{H}$. Non-invasive magnetic resonance imagingguided focused ultrasound treatment for uterine fibroids - early experience. Eur J Obstet Gynaecol Reprod Biol. 2008; 139(2):199-203.

12. Rabinovici J, Stewart EA. New interventional techniques for adenomyosis. Best Pract Res Clin Obstet Gynaecol. 2006; 20(4):617-36.

13. Stewart EA, Rabinovici J, Tempany CMC, Inbar $Y_{\text {, }}$ Regan L, Gastout B, et al. Clinical outcomes of focused ultrasound surgery for the treatment of uterine fibroids. Fertil Steril. 2006; 85(1):22-9.

14. Yoon S-W, Kim KA, Cha SH, Kim YM, Lee C, Na Y-J, et al. Successful use of magnetic resonance-guided focused ultrasound surgery to relieve symptoms in a patient with symptomatic focal adenomyosis. Fertil Steril. 2008; 90(5):2018.e13-.e15.

15. Hindley J, Gedroyc WM, Regan L, Stewart E, Tempany C, Hynnen K, et al. MRI guidance of focused ultrasound therapy of uterine fibroids: Early results. Am J Roentgenol. 2004; 183(6):1713-9.

16. Hesley G, Gorny K, Woodrum D. MR elastography following focused ultrasound treatment of uterine fibroids. J Ther Ultrasound. 2014; 2(Suppl 1):A13-A.

17. Ikink ME, Voogt MJ, Bosch MAAJ, Nijenhuis RJ, Keserci B, Kim Y-s, et al. Diffusion-weighted magnetic resonance imaging using different $b$-value combinations for the evaluation of treatment results after volumetric MR-guided high-intensity focused ultrasound ablation of uterine fibroids. Eur Radiol. 2014; 24(9):2118-27.

18. Jacobs MA, Herskovits EH, Kim HS. Uterine fibroids: Diffusion-weighted MR imaging for monitoring therapy with focused ultrasound surgery-preliminary study. Radiology. 2005; 236(1):196-203.

19. Jacobs MA, Gultekin DH, Kim HS. Comparison between diffusion-weighted imaging, $T(2)$-weighted, and postcontrast $\mathrm{T}(1)$-weighted imaging after MRguided, high intensity, focused ultrasound treatment of uterine leiomyomata: Preliminary results. Med Phys. 2010; 37(9):4768-76.

20. Jacobs MA, Ouwerkerk R, Kamel I, Bottomley PA, Bluemke DA, Kim HS. Proton, diffusion-weighted imaging, and sodium (23Na) MRI of uterine leiomyomata after MR-guided high-intensity focused ultrasound: A preliminary study. J Magn Reson Imaging. 2009; 29(3):649-56.

21. Liapi E, Kamel IR, Bluemke DA, Jacobs MA, Kim HS. Assessment of response of uterine fibroids and myometrium to embolization using diffusionweighted echoplanar MR imaging. J Comput Assist Tomogr. 2005; 29(1):83-6.

22. Pilatou MC, Stewart EA, Maier SE, Fennessy FM, Hynynen K, Tempany CMC, et al. MRI-based thermal dosimetry and diffusion-weighted imaging of MRI-guided focused ultrasound thermal ablation of uterine fibroids. J Magn Reson Imaging. 2009; 29(2):404-11.

23. Voogt MJ, Keserci B, Kim YS, Rhim H, Lim HK, Mougenot $\mathrm{C}$, et al. Diffusion weighted MR imaging to evaluate treatment results after volumetric MR-guided high intensity focused ultrasound of uterine fibroids: Influence of different b-values. AIP Conference Proceedings. 2011; 1359(1):483-7.

24. Abdullah BJJ, Subramaniam RV, Omar SS, Wragg P, Ramli N, Wui AL, et al. Magnetic resonance-guided focused ultrasound surgery (MRgFUS) treatment for uterine fibroids. Biomed Imaging Interv J. 2010; 6(2):e15. 
25. Dietrich O, Biffar A, Baur-Melnyk A, Reiser MF. Technical aspects of MR diffusion imaging of the body. Eur J Radiol. 2010; 76(3):314-22. 